\title{
Single Molecule Photobleaching Probes the Exciton Wave Function in a Multichromophoric System
}

\author{
J. Hernando, ${ }^{1}$ J. P. Hoogenboom, ${ }^{1}$ E. M. H. P. van Dijk, ${ }^{1}$ J. J. García-López,${ }^{2}$ M. Crego-Calama, ${ }^{2}$ D. N. Reinhoudt, ${ }^{2}$ \\ N. F. van Hulst, ${ }^{1}$ and M. F. García-Parajo ${ }^{1, *}$ \\ ${ }^{1}$ Applied Optics group, Faculty of Science \& Technology and MESA ${ }^{+}$Institute for Nanotechnology, University of Twente, \\ P.O. Box 217, 7500 AE Enschede, The Netherlands \\ ${ }^{2}$ Supramolecular Chemistry \& Technology group, Faculty of Science \& Technology and MESA ${ }^{+}$Institute for Nanotechnology, \\ University of Twente, P.O. Box 217, 7500 AE Enschede, The Netherlands
}

(Received 24 April 2004; published 2 December 2004)

\begin{abstract}
The exciton wave function of a trichromophoric system is investigated by means of single molecule spectroscopy at room temperature. Individual trimers exhibit superradiance and loss of vibronic structure in emission spectrum, features proving exciton delocalization. We identify two distinct photodegradation pathways for single trimers upon sequential photobleaching of the chromophores. The rate of each pathway is a measure for the contribution of the separate dyes to the collective excited state of the system, in this way probing the wave function of the delocalized exciton.
\end{abstract}

DOI: 10.1103/PhysRevLett.93.236404

PACS numbers: 71.35.Aa, 34.30.+h, 34.50.Gb

The optical behavior of nearby quantum emitters is governed by Coulombic interactions, giving rise to transfer or even delocalization of the electronic excitation energy upon irradiation [1,2]. Understanding the energy flow in such systems is a key step towards the design of multichromophoric assemblies for applications in molecular photonics and electronics. In recent years, single molecule spectroscopy (SMS) has proven a powerful technique to investigate natural [3-5] as well as synthetic [6-14] multichromophoric complexes.

Until now SMS experiments have mainly been devoted to the investigation of incoherent energy transfer in weakly coupled systems, and only recently a study of a strongly interacting dimer at room temperature has been reported [14]. Because of the large degree of chromophoric coupling in this type of systems, collective excited states are expected where the electronic excitation energy is coherently delocalized (delocalized exciton) [1]. In this Letter we report on the probing of the wave function of such delocalized exciton. We have used single molecule fluorescence spectroscopy at room temperature to study a linear trichromophoric assembly, the simplest system where distinct contributions of the chromophores to the collective exciton state are predicted. We have analyzed the signatures in the emission of individual trimers that are indicative of exciton delocalization. This has allowed us to uncover different photodegradation pathways upon sequential chromophore photobleaching. The rates for these pathways reflect the contribution of each separate dye to the delocalized exciton wave function.

The molecular trimer investigated was prepared by directly linking three tetraphenoxy-perylene diimide molecules in a parallel head-to-tail configuration. Quantum-mechanical calculations show that the resulting trimer (TPD) has a rigid structure with the individual perylene units being perpendicular to each other, thus preventing full extension of the $\pi$-conjugation path throughout the system (Fig. 1). On the other hand, the short interchromophoric distance $(1.3 \mathrm{~nm})$ and the alignment of the dipoles in a parallel head-to-tail configuration guarantee strong dipolar interaction between nearby chromophores $\left(J=-300 \mathrm{~cm}^{-1}\right)[1,2]$.

Single molecule experiments were performed on thin film samples prepared by spin coating a toluene solution of TPD $\left(10^{-9} \mathrm{M}\right)$ and poly(methyl methacrylate) $(5 \mathrm{~g} / \mathrm{l})$ onto a glass substrate. The emission arising from single perylene trimers was investigated using a homebuilt confocal optical microscope allowing for simultaneous fluorescence intensity, polarization, lifetime, and spectral measurements [13]. Single TPD molecules were excited with 280 fs pulses at $\lambda=568 \mathrm{~nm}$ (circularly polarized light), a repetition rate of $8 \mathrm{MHz}$, and a mean power density of $2.5 \mathrm{~kW} \mathrm{~cm}^{-2}$. Antibunching experiments were performed using a cw laser at $\lambda=568 \mathrm{~nm}$ (circularly polarized light) and a power density of $7 \mathrm{~kW} \mathrm{~cm}^{-2}$, where the fluorescence emission was detected in a HanburyBrown-Twiss scheme [15].

Figure 2(a) depicts the time evolution of the fluorescence intensity for a single TPD molecule (molecule 1).

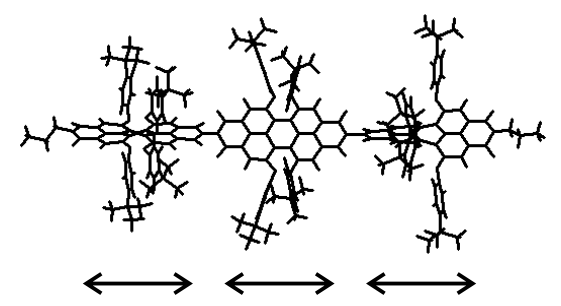

FIG. 1. Optimal geometry of TPD calculated by means of the semiempirical MNDO (modified neglect of differential overlap) method [19]. The arrows show the orientation of the transition dipole moments of the perylene units in the trimer. 

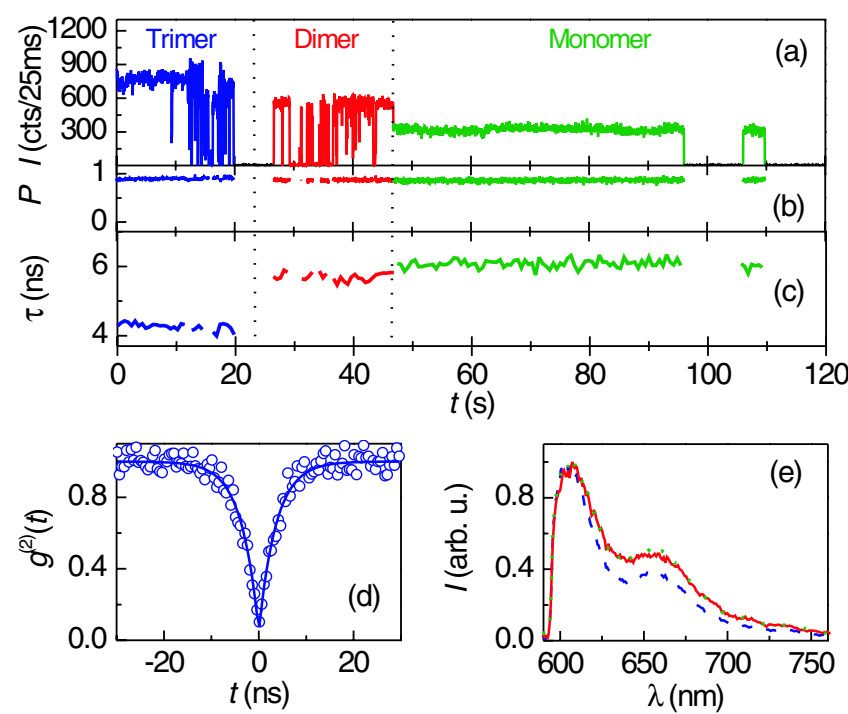

FIG. 2 (color online). (a)-(c) Fluorescence intensity (I), polarization $(P)$, and lifetime $(\tau)$ trajectories recorded for a single TPD molecule (molecule 1). Dashed lines separate trimer, dimer, and monomer levels. $P$ is defined as $P=\left(I_{x}-I_{y}\right) /\left(I_{x}+\right.$ $I_{y}$ ), where $I_{x}$ and $I_{y}$ are the emission components detected in two orthogonal polarization directions. Each $\tau$ value derives from a monoexponential fitting to the photon arrival time decay built every $600 \mathrm{~ms}$. (d) Experimental (symbols) and fitted (lines) second-order correlation function for the trimer level of a TPD molecule. $\tau=3.7 \mathrm{~ns}$ is recovered from the fitting. (e) Emission spectra of the trimer (dashed line), dimer (solid line), and monomer (dotted line) levels of molecule 1.

Three different intensity levels above background are identified, a situation found for $66 \%$ of the trimers. These distinct levels arise from stepwise photodegradation of the perylene units in the trimer, a behavior characteristic for multichromophoric systems [3,5-8,10-14]. Thus, emission from the TPD molecule with three, two, and one intact chromophores is responsible for the high (trimer), intermediate (dimer), and low (monomer) intensity levels, respectively. Figure 2(b) shows that no variations in fluorescence polarization occur between intensity levels. Aside from the absence of conformational changes upon photobleaching of the chromophores, this result demonstrates a parallel arrangement of the transition dipole moments of the interacting dye units, as supported by our calculations of the TPD structure. Consequently, the intensity ratio for the different levels in Fig. 2(a) (2.6:1.8:1) should match the value expected a priori for a system with three identical in-plane dyes (3:2:1), provided that the absorption cross section $\left(\sigma_{\text {abs }}\right)$ scales with the number of chromophores and the emission quantum yield $\left(\Phi_{f}\right)$ remains invariant. Bulk experiments on toluene solutions of tetraphenoxy-perylene diimide monomer, dimer, and trimer confirm $\Phi_{f \text {,trimer }} \simeq \Phi_{f \text {,dimer }} \simeq$ $\Phi_{\text {f,monomer }} \simeq 1$, while $\sigma_{\text {abs,trimer }}$ and $\sigma_{\text {abs,dimer }}$ are slightly lower than 3 and 2 times $\sigma_{\text {abs,monomer }}$ at $\lambda=568 \mathrm{~nm}$, respectively. The latter accounts for the small deviation found with respect to the ideal 3:2:1 intensity ratio value.
Coupling effects in the emission of the trimer are unambiguously proven by the interphoton time distribution shown in Fig. 2(d) for the trimer level of a single TPD molecule, which follows the second-order correlation function $g^{(2)}(t)$ at short times. A pronounced dip at $t=$ $0\left[g^{(2)}(0)=0.11\right]$ is observed (photon antibunching), in contrast to what is expected for three in-plane identical dyes emitting independently $\left[g^{(2)}(0)=0.67\right]$. This demonstrates that the trimer fluorescence arises from a single emitting site at a time, a behavior expected for both weakly $[8,10]$ and strongly [9] coupled molecules.

Figure 2(e) displays the total fluorescence emission spectra for the different levels of molecule 1. Spectral changes between levels are observed; namely, the contribution of the vibronic band at $\lambda=660 \mathrm{~nm}$ rises upon sequential photobleaching. The intensity ratio between the two vibronic peaks in the spectra is equal to 2.6, 2.1, and 1.9 for the trimer, dimer, and monomer levels, respectively. This effect is consistent with exciton delocalization in the system, leading to a weaker coupling between electronic and vibrational motions and, consequently, smoothing out the vibronic features in the spectrum [2]. A similar phenomenon has recently been reported for single perylene dimers at room temperature [14]. For a linear arrangement of the interacting dipoles ( $J$ aggregate), exciton delocalization should also be accompanied by a redshift in the emission spectrum $[1,2]$. On average over all investigated molecules we found a mean displacement between trimer and monomer emission maxima of $\sim-95 \mathrm{~cm}^{-1}$. This is in fair agreement with the ensemble solution result of $\sim-215 \mathrm{~cm}^{-1}$, if one takes into consideration the chemical difference between the perylene monomer and the monomer level of a partially photobleached trimer. A larger spectral shift $\left(\sim-440 \mathrm{~cm}^{-1}\right)$ is predicted by exciton theory; however, in practice it is counterbalanced to a significant extent by the spread in energies of the perylene units in the polymer matrix at room temperature (static disorder, $\sigma_{d} \sim$ $320 \mathrm{~cm}^{-1}$ for the monomer level of 25 single trimers). Ultimately, disorder may cause the exciton shift to fall below our spectral sensitivity $\left(\sim 40 \mathrm{~cm}^{-1}\right)$, as observed for molecule 1.

Figure 2(c) shows the time evolution of the fluorescence excited state lifetime for molecule 1 , which displays interlevel variations $(\langle\tau\rangle=4.3,5.7$, and $6.1 \mathrm{~ns}$ for the trimer, dimer, and monomer levels, respectively). Together with constant $\Phi_{f}$, this result provides a clear signature of strong coupling in the system and confirms exciton delocalization. In $J$ aggregates the electronic transition from the ground state to the lowest exciton level carries nearly all oscillator strength of the system, resulting in an enhancement of the radiative rate (superradiance) and thus, a decrease of the excited state lifetime $[1,2]$. This constitutes one of the first demonstrations of such a phenomenon in a multichromophoric assembly at the single molecule level under ambient conditions [14]. 
In what follows we focus on the analysis of superradiance, a direct fingerprint of exciton delocalization herein used to report on the exciton wave function of the TPD molecule.

Figures 3(a)-3(c) show the fluorescence lifetime histograms for the different intensity levels of 121 trimers. Their width results from heterogeneity of the local environment, i.e., the polymer host. In spite of this, the lifetime distributions clearly shift to lower values of $\tau$ with the number of interacting chromophores. Thus, $\langle\tau\rangle=4.1$, 5.3, and $5.9 \mathrm{~ns}$ for the trimer, dimer, and monomer levels. Figure $3(\mathrm{~d})$ shows the distribution of the $\tau_{\text {monomer }} / \tau_{\text {trimer }}$ ratio for the molecules depicted in Figs. 3(a)-3(c). As $\Phi_{f \text {,trimer }} \simeq \Phi_{f \text {,monomer }} \simeq 1$, the lifetime ratio yields the degree of superradiance for each single trimer, providing a direct measure of its coupling strength [14]. A broad distribution with a mean value equal to 1.5 is obtained, in contrast to the prediction of $\tau_{\text {monomer }} / \tau_{\text {trimer }}=2.9$ for TPD in absence of disorder [1]. At room temperature in a polymer matrix, however, both static (dye energy fluctuations) and dynamic (exciton-phonon coupling) disorder play an important role and may result in partial break down of the exciton delocalization [16], as reported for individual perylene dimers [14]. The effect of disorder accounts for our results and suggests intermediate exciton coupling under the current experimental conditions. The spread in chromophoric site energies also accounts for coupling strength variations from molecule to molecule [14], as proven by the width of the $\tau_{\text {monomer }} / \tau_{\text {trimer }}$ histogram. Interestingly, those molecules showing higher lifetime ratios in Fig. 3(d) also display larger spectral variations, demonstrating that both types of properties are equally affected by disorder [17].

In contrast to the behavior of the trimer and monomer levels, the dimer fluorescence lifetime distribution in Fig. 3(b) is bimodal, showing two distinct peaks at 4.8
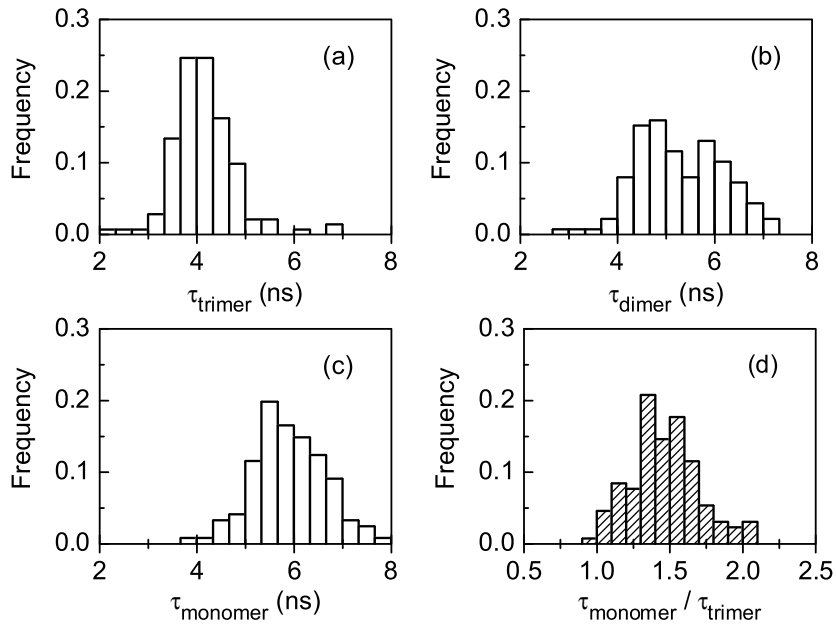

FIG. 3. (a)-(c) Fluorescence lifetime distributions measured for the trimer, dimer, and monomer levels of 121 TPD molecules, respectively. Each $\tau$ value determined has an accuracy of 0.1 ns. (d) Histogram of the $\tau_{\text {monomer }} / \tau_{\text {trimer }}$ ratio. and $5.9 \mathrm{~ns}$. The value of $4.8 \mathrm{~ns}$ lies between those obtained for the trimer (4.1 ns) and monomer (5.9 ns) species and compares well with findings on tetraphenoxy-perylene diimide dimers $(4.8 \mathrm{~ns})$. On the other hand, the value of $5.9 \mathrm{~ns}$ of the second peak is similar to $\left\langle\tau_{\text {monomer }}\right\rangle$. This intricate situation is due to the existence of two different subpopulations of dimer species arising from two distinct photodegradation pathways of TPD, as sketched in Fig. 4(a). In pathway 1 one of the perylenes at the end of the trimer ( $S 1$ and $S 2)$ bleaches, yielding dimer molecules with a short interchromophoric distance $(1.3 \mathrm{~nm})$ and displaying coherent exciton effects, i.e., superradiance. In pathway 2 the central chromophore $(C)$ photodegradates and the distance between the two intact dye units becomes larger $(2.6 \mathrm{~nm})$. In this case the coupling strength is decreased by an order of magnitude, resulting in weak exciton effects (Förster-type of energy transfer). Consequently, this subpopulation of dimer species shows monomerlike emission properties.

In order to discriminate between the two types of dimer species, the correlation between $\tau_{\text {dimer }} / \tau_{\text {trimer }}$ and $\tau_{\text {monomer }} / \tau_{\text {dimer }}$ ratios for all trimers is plotted in Fig. $4(\mathrm{~b})$. Heterogeneity in coupling strength under the current experimental conditions smears out the separation in lifetime ratios expected for the two types of dimer levels, making them overlap for values of $\tau_{\text {dimer }} / \tau_{\text {trimer }}$ and $\tau_{\text {monomer }} / \tau_{\text {dimer }}$ close to unity. TPD molecules yielding lifetime ratios in this region are those with weaker collective effects and have been excluded from further analysis. When concentrating on individual trimers with

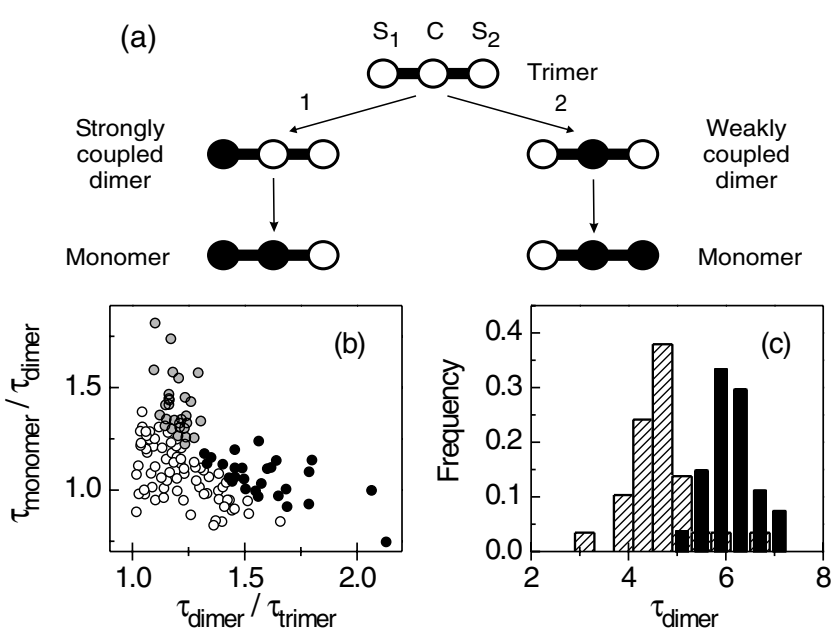

FIG. 4. (a) Sketch of the two bleaching pathways for TPD. Empty and full circles stand for intact and bleached dye units. (b) $\tau_{\text {monomer }} / \tau_{\text {dimer }}$ vs $\tau_{\text {dimer }} / \tau_{\text {trimer }}$ correlation plot for $121 \mathrm{TPD}$ aggregates. Molecules with low coupling strength are shown as empty circles $\left(\tau_{\text {monomer }} / \tau_{\text {trimer }}<1.5\right.$ ). Two distinct sets of molecules appear visible for trimers with stronger interaction $\left(\tau_{\text {monomer }} / \tau_{\text {trimer }}>1.5\right)$, which are associated with bleaching pathways 1 (gray circles) and 2 (black circles). (c) Lifetime distributions corresponding to the dimer levels originated from pathways 1 (crossed bars) and 2 (full bars). 
coupling strength above the average value $\left[\tau_{\text {monomer }} /\right.$ $\tau_{\text {trimer }}>1.5$; cf. Fig. 3(d)], two separate groups of molecules become apparent in Fig. 4(b), which are associated with distinct photodegradation processes. The set of data with $\tau_{\text {monomer }} / \tau_{\text {dimer }} \sim 1$ should correspond to dimer species arising from pathway 2, while the data at larger values of $\tau_{\text {monomer }} / \tau_{\text {dimer }}$ are ascribed to trimers undergoing pathway 1 . Although the properties of the trimer and monomer levels of these two sets of data are similar $\left(\left\langle\tau_{\text {trimer }}\right\rangle=3.9 \mathrm{~ns}\right.$ in both cases; $\left\langle\tau_{\text {monomer }}\right\rangle=6.1$ and $6.3 \mathrm{~ns}$ ), their dimer levels are markedly different, as shown in Fig. 4(c). Whereas the dimer lifetime distribution for TPD molecules undergoing pathway 2 resembles that of the monomer $[\langle\tau\rangle=6.1 \mathrm{~ns}$; cf. Fig. 3(c)], dimers arising from pathway 1 display shorter $\tau$ values $(\langle\tau\rangle=$ $4.7 \mathrm{~ns})$, thus proving exciton delocalization. Certainly, the criterion used to separate the two dimer species allows us to fully uncover the bimodal feature of the overall $\tau_{\text {dimer }}$ distribution. As expected, this feature was not observed for the weakly coupled trimers $\left(\tau_{\text {monomer }} / \tau_{\text {trimer }}<1.5\right)$, which present a unimodal lifetime distribution.

The populations of the two sets of molecules distinguished in Fig. 4(c) are rather similar (29 stronger vs 27 weaker coupled dimers), indicating that equal amounts of strongly coupled trimers photodegradate according to either pathways 1 or 2 (ratio path. $1 /$ path. $2=1.1 \pm 0.3$ ). The same result is also obtained when fitting the bimodal lifetime distribution for the dimer species in Fig. 3(b) with a double Gaussian function. Although the understanding of dye photodegradation processes is still limited $[4,18]$, bleaching appears as a photodriven phenomenon whose probability depends on the excitation rate of the system and the chance of the subsequently photoinduced states to react and yield a nonradiative species. Therefore, the photodegradation rates for individual dyes in multichromophoric systems should report on the evolution of the excited state energy in the system.

For a parallel head-to-tail trimer with three equivalent independent emitters, a pure statistical degradation probability of the chromophores is expected (ratio path. $1 /$ path. $2=2$ ), the bleaching chance being twice as high for the side dyes together than for the central one. The same result is obtained in the case of incoherent energy transfer due to weak chromophoric coupling. Clearly, our experimental bleaching ratio is significantly lower.

In the absence of disorder the wave function of the delocalized exciton over the strongly coupled trimer is [1]

$$
|\Psi\rangle=0.51\left|\psi_{S 1}\right\rangle+0.69\left|\psi_{C}\right\rangle+0.51\left|\psi_{S 2}\right\rangle,
$$

where $\psi_{S 1}, \psi_{C}$, and $\psi_{S 2}$ denote the states of the system in which $S 1, C$, and $S 2$ are excited, respectively. To derive Eq. (1) nearest-neighbor and long-range interactions have been taken into account. If the square of the coefficients in (1) is taken, the state where the central dye carries the molecular excitation is found to contribute largely to the collective excited state (48\%), counterbalancing the higher population of side chromophores and leading to a bleaching ratio path. $1 /$ path. $2=1.1$. To account for disorder, we performed numerical simulations of (1) for a population of dyes with their energy within a Gaussian distribution of $\sigma_{d}=320 \mathrm{~cm}^{-1}$ in width. Even assuming such an amount of static disorder, only a slightly reduced contribution of $\psi_{C}$ to the overall wave function was found (43\%), leading to an average 1.3 bleaching ratio. Our experimental $1.1 \pm 0.3$ bleaching ratio is in good agreement with these values, thus demonstrating that the photodamage probabilities measured for TPD properly describe the contribution of each chromophore to the collective excited state of the system. Hence, we conclude that single molecule photobleaching probes the coefficients of the wave function of the delocalized exciton in a multichromophoric assembly.

This work has been supported by the EC Program IHP99 (HPMF-CT-2002-01698) and MCyT (BQU200204269-C02-02) (J. H.), VW-Stiftung (J.P. H.), FOM (E. M. H. P. v. D.), and KNAW (M. F. G. P.).

*Electronic address: m.f.garciaparajo@tnw.utwente.nl

[1] A.S. Davydov, in Theory of Molecular Excitons (Plenum, New York, 1971).

[2] M. Kasha, H. R. Rawls, and M. Ashaf El-Bayoumi, Pure Appl. Chem. 11, 371 (1965).

[3] A. M. van Oijen et al., Science 285, 400 (1999); C. Hofmann et al., Phys. Rev. Lett. 90, 013004 (2003).

[4] L. Ying and X.S. Xie, J. Phys. Chem. B 102, 10399 (1998).

[5] M. F. García-Parajó et al., Proc. Natl. Acad. Sci. U.S.A. 98, 14392 (2001).

[6] D. A. Vanden Bout et al., Science 277, 1074 (1997); J. G. Müller et al., Phys. Rev. Lett. 91, 267403 (2003).

[7] J. Hofkens et al., J. Am. Chem. Soc. 122, 9278 (2000); M. Cotlet et al., J. Am. Chem. Soc. 125, 13609 (2003).

[8] P. Tinnefeld et al., J. Am. Chem. Soc. 124, 14310 (2002).

[9] C. Hettich et al., Science 298, 385 (2002).

[10] T. Christ et al., J. Lumin. 98, 23 (2002); C. G. Hubner et al., Phys. Rev. Lett. 91, 093903 (2003).

[11] J. Hofkens et al., Proc. Natl. Acad. Sci. U.S.A. 100, 13146 (2003).

[12] R. C. Liu et al., J. Phys. Chem. A 107, 6522 (2003).

[13] J. Hernando et al., J. Phys. Chem. A 107, 43 (2003).

[14] M. Lippitz et al., Phys. Rev. Lett. 92, 103001 (2004).

[15] R. Hanbury-Brown and R. Twiss, Nature (London) 177, 27 (1956).

[16] F. C. Spano, J. R. Kuklinski, and S. Mukamel, Phys. Rev. Lett. 65, 211 (1990); M. Bednarz, V. A. Malyshev, and J. Knoester, Phys. Rev. Lett. 91, 217401 (2003).

[17] J. Hernando et al. (to be published).

[18] T. Christ et al., Angew. Chem., Int. Ed. Engl. 40, 4192 (2001).

[19] M. J. Frisch et al., GAUSSIAN 98, Revision A.7, Gaussian, Inc., Pittsburgh, PA, 1998. 